\title{
Treatment Sequencing Patterns in Patients with
} Metastatic Urothelial Cancer Treated in the

\section{Community Practice Setting in the United States: SPEAR-Bladder (Study informing treatment Pathway dEcision in bladder cAnceR)}

This article was published in the following Dove Press journal: ClinicoEconomics and Outcomes Research

\author{
Gurjyot K Doshi' \\ Abhijeet Bhanegaonkar ${ }^{2}$ \\ Mairead Kearney ${ }^{3}$ \\ Murtuza Bharmal ${ }^{2}$ \\ Paul Cislo ${ }^{4}$ \\ Ruth $\mathrm{Kim}^{4}$ \\ Marley Boyd ${ }^{5}$ \\ Kathleen M Aguilar (1D ${ }^{5}$ \\ Hemant Phatak ${ }^{2}$ \\ 'Texas Oncology, Houston, TX, USA; \\ ${ }^{2} E M D$ Serono Research \& Development \\ Institute, Inc., Rockland, MA, USA; an \\ affiliate of Merck KGaA, Darmstadt, \\ Germany, ${ }^{3}$ Merck KGaA, Darmstadt, \\ Germany; ${ }^{4}$ Pfizer Inc., New York, NY, \\ USA; ${ }^{5}$ Data, Evidence and Insights, \\ McKesson Life Sciences, The US \\ Oncology Network, The Woodlands, \\ TX, USA
}

Purpose: Clinical trial evidence has affirmed the role for immuno-oncology (IO) treatment for locally advanced or metastatic urothelial carcinoma (la/mUC). This Study informing treatment Pathway dEcision in bladder cAnceR (SPEAR-Bladder) aimed to provide insight into the optimal sequencing of IO treatments among la/mUC patients treated in the US Oncology Network.

Patients and Methods: This was a retrospective analysis of adult patients with $1 \mathrm{a} / \mathrm{mUC}$ who initiated first-line chemotherapy followed by either IO therapy (C-IO subgroup) or chemotherapy (C-C subgroup) between 01/01/2015 and 04/30/2017 and included a potential follow-up period through 06/30/2017. Data were sourced from iKnowMed electronic health records. Patient and treatment characteristics were assessed descriptively, with Kaplan-Meier methods used to evaluate time-to-event outcomes, including overall survival (OS).

Results: A total of 117 patients were included in this analysis (median age 69 years, 74.4\% male, 88.0\% Caucasian): 79 and 38 patients were in the C-IO and C-C subgroups, respectively. The median OS was 19.2 months among patients who received the C-IO sequence and 11.9 months among those who received the $\mathrm{C}-\mathrm{C}$ treatment sequence.

Conclusion: These results suggest that patients who received the $\mathrm{C}-\mathrm{IO}$ treatment sequence had notable improvement in OS compared with those who received the $\mathrm{C}$-C sequence. In light of the rapidly evolving therapeutic landscape, further investigation will be required to determine how best to select the optimal therapeutic regimen and sequencing for patients with la/mUC.

Keywords: urothelial carcinoma, SPEAR-Bladder, real-world clinical outcomes, treatment sequencing, retrospective, community oncology setting

\section{Introduction}

In treatment-naïve patients with confirmed locally advanced or metastatic urothelial carcinoma (la/mUC), platinum-based chemotherapy is the standard of care in the first-line (1L) setting. ${ }^{1,2}$ For patients who are ineligible for cisplatin, gemcitabinecarboplatin combination chemotherapy, with a lower toxicity profile, has been the mainstay treatment option. ${ }^{3}$ However, clinical management of la/mUC is challenged by heterogenous patient populations and the need to optimally sequence
Correspondence: Abhijeet Bhanegaonkar Director, US Health Economics and Outcomes Research - Oncology, North America Medical Affairs, EMD Serono, Inc., One Technology Place, Rockland, MA 02370 , USA

Tel + $78|-68|-2483$

Email abhijeet.bhanegaonkar@emdserono. com 
treatments to yield durable responses. ${ }^{4}$ Treatment with chemotherapy is associated with median overall survival (OS) estimates of 9 to 15 months. ${ }^{2,5,6}$ Following relapse on platinum-based chemotherapy, median OS is estimated to be 5 to 7 months. ${ }^{2,7}$ Alternative treatment strategies have recently emerged, including immuno-oncology (IO)-based regimens that improve response rates and survival. ${ }^{2,4,8,9}$

For patients who progress following $1 \mathrm{~L}$ chemotherapy treatment, IO-based regimens have emerged as the standard of care. ${ }^{3}$ IO therapies are checkpoint inhibitors that target programmed cell death 1 ligand 1 (PD-L1) or its receptor (programmed cell death 1 protein [PD-1]). PD-L1 is an immune inhibitory molecule that, when expressed, can bind to $\mathrm{T}$ lymphocytes and reduce the ability of the host's immune system to respond to the tumor. ${ }^{10}$ The therapeutic potential of agents that target the PD-1/PDL1 pathway has been reported in multiple studies for the treatment of advanced cancer. ${ }^{11}$ For UC, studies have found that approximately $20 \%$ of these tumors have a positive PD-L1 expression in the tumor membrane. ${ }^{12,13}$

To date, 5 IO therapies have been approved by the US Food and Drug Administration (FDA) for the treatment of la/mUC following progression on platinum-based therapy: atezolizumab (a PD-L1 inhibitor), avelumab (a PD-L1 inhibitor), durvalumab (a PD-L1 inhibitor), nivolumab (a PD-1 inhibitor) and pembrolizumab (a PD-1 inhibitor). ${ }^{3,4}$ Based on initial clinical trial results, each of these therapies is associated with favorable efficacy and safety profiles. $^{2,4,8,9}$

Based upon the results from the Phase III JAVELIN Bladder 100 trial (NCT02603432), avelumab was approved by the US FDA in June 2020 for use in patients with $1 \mathrm{a} / \mathrm{mUC}$ that has not progressed with $1 \mathrm{~L}$ platinumcontaining chemotherapy, making it the first and only FDA-approved maintenance therapy for such patients. ${ }^{14}$ With this approval and incorporation into treatment guidelines, platinum-containing chemotherapy regimens followed by $1 \mathrm{~L}$ avelumab maintenance therapy is a preferred treatment option for the treatment of $\mathrm{la} / \mathrm{mUC}$ in patients whose disease does not progress with $1 \mathrm{~L}$ platinum-containing chemotherapy. ${ }^{3,15}$ The JAVELIN Bladder 100 trial evaluated avelumab plus best supportive care versus best supportive care alone as $1 \mathrm{~L}$ maintenance among la/mUC patients whose disease did not progress with 1L platinum-based chemotherapy. ${ }^{14,16-18}$ At the planned interim analysis, Powles et al (2020) reported that the JAVELIN Bladder 100 study met its primary endpoint of significantly prolonging OS in the study's two primary populations: the overall population and the PD-L1+ population. ${ }^{14,18}$

With the advent of IO-based therapies, the treatment landscape for la/mUC shifted considerably, and limited studies have examined how these trends manifested in the real-world setting. The aim of SPEAR-Bladder was, therefore, to provide a reflection on the treatment patterns during the pre-IO period as well as insights into the early adoption of IO therapies into the community oncology setting. Particular focus was given to how treatment sequences influence OS.

\section{Patients and Methods Study Design, Data Source, and Patient Population}

This was a retrospective, observational, descriptive study of US patients with la/mUC who had evidence of initiating treatment with either a systemic chemotherapy or IO therapy (as $1 \mathrm{~L}$ through third line [3L] of therapy) between 01 January 2015 and 30 April 2017 (ie, patient identification period) in the US Oncology Network (USON). Eligible patients were followed through 30 June 2017. The USON includes 1400 affiliated physicians operating in over 450 sites of care across states and provides care for nearly 1 million cancer patients annually. ${ }^{19}$

The study protocol was granted an exception and waiver of informed consent by the US Oncology Institutional Review Board. Waiver of informed consent was granted by the US Oncology Institutional Review Board as a deidentified dataset and stringent precautions were taken to reduce risk of reidentification. As such, this study was deemed to have minimal risk to patients. Additionally, it was not feasible to obtain informed consent from eligible patients or provide them with additional information about their participation, as this was a retrospective study of a critically ill population, many of whom were deceased or had not received further care within the USON prior to initiation of the research.

Focus was given to the subset of patients who received the two most commonly observed $1 \mathrm{~L}$ through $3 \mathrm{~L}$ treatment sequences: 1L chemotherapy followed by second-line (2L) IO-based regimens without $3 \mathrm{~L}$ treatment (C-IO subgroup), and $1 \mathrm{~L}$ chemotherapy followed by $2 \mathrm{~L}$ chemotherapy without 3L treatment (C-C subgroup). Results of the broader study population have been reported previously. ${ }^{20,21}$

Most study data were sourced from the USON's electronic health record (EHR) system, iKnowMed (iKM; 
McKesson Corporation, Las Colinas, TX, USA), with death dates sourced from both iKM and the Social Security Administration Death Master File (DMF).

Eligible patients received at least 2 cycles of therapy during the patient identification period and had at least 2 follow-up visits at a USON clinic utilizing the full EHR capacities of $\mathrm{iKM}$ and whose data were accessible for research purposes. Patients enrolled in clinical trials and those with other cancer diagnoses were excluded.

Baseline variables were assessed for the 30-day period prior to treatment initiation. All study variables and outcomes were assessed regardless of maximum follow-up, using data available until the end of the study observation period (30 June 2017); no minimum follow-up period was required.

\section{Statistical Methods}

Descriptive analyses were conducted to assess demographic, clinical, and treatment characteristics. Computational algorithms were applied to categorize therapy sequences across treatment lines based on start and stop dates as well as the provider-defined line of therapy indicator in iKM. Categorical variables (eg, gender, performance status) were reported as frequencies and percentages. Continuous variables such as age were reported as mean, standard deviation, median, and range. Time to $2 \mathrm{~L}$ was defined as the time from initiation of $1 \mathrm{~L}$ treatment to initiation of $2 \mathrm{~L}$ treatment.

Descriptive analyses were performed to assess associations between categorical and continuous variables. Specifically, Chi-square testing was used to assess associations between categorical variables when patient counts for single cells within the results tables were greater or equal to 5. When distribution could not be assumed to be Chi-square, then the Fisher's exact test was used. Depending on normality, ANOVA/t-tests or KruskalWallis tests were used for continuous variables.

Kaplan-Meier curves and Cox proportional hazard models were constructed to illustrate time-to-event outcomes with medians and 95\% confidence intervals (CIs) and stratified to present results by the most common treatment sequences. Treatment sequence groups were compared using unadjusted hazard ratios (HR) and HR p-values. Time to treatment failure (TTF) was defined as the interval (in months) between treatment initiation and discontinuation of 1L chemotherapy. Treatment-free interval (TFI) was defined as the interval (in months) between the last administration of $1 \mathrm{~L}$ therapy and the start date of subsequent treatment. OS was defined as the interval (in months) between the start of treatment until the date of death (any cause) as documented in the DMF or the iKM EHR database. For all Kaplan-Meier time-to-event analyses, patients who did not die within the study observation period were censored at the study end date or the last visit date available in the dataset, whichever occurred first.

\section{Results \\ Study Population}

In total, 502 patients initiated a qualifying treatment in the 1L, 2L or 3L setting: 430 patients were followed from initiation of $1 \mathrm{~L}$ treatment, 66 from initiation of $2 \mathrm{~L}$ treatment, and 6 from initiation of 3L treatment (Figure 1). Following 1L treatment discontinuation, 152 (35.3\%) patients proceeded to $2 \mathrm{~L}$ treatment. Of the patients who proceeded from $1 \mathrm{~L}$ to $2 \mathrm{~L}$ treatment during the study observation period, 131 did so in less than 6 months, 17 did so within 6 to 12 months and 4 after 12 months. $^{21}$ Compared to the 278 patients who did not proceed, those who received $2 \mathrm{~L}$ treatment were younger (median age 68 vs 74 years; $\mathrm{P}<0.0001)$, had more advanced disease at diagnosis (59.8\% vs $47.1 \%$ with stage III/IV, respectively; $\mathrm{P}=0.0127)$ and had a higher number of documented metastatic sites (34.9\% vs $17.6 \%$, respectively; $\mathrm{P}=0.0256)$.

The most common treatment sequences were $1 \mathrm{~L}$ chemotherapy followed by either IO-based regimens (C-IO subgroup) or chemotherapy (C-C subgroup), without a subsequent treatment (Figure 1). In total, 79 (18.4\%) patients received the $\mathrm{C}$-IO treatment sequence and 38 (8.8\%) the C-C treatment sequence.

The distribution of the most common treatment regimens received by the study population by line of therapy is presented in Figure 2.

\section{Patient Characteristics}

Across the $\mathrm{C}-\mathrm{IO}$ and $\mathrm{C}-\mathrm{C}$ subgroups, the median age was 69 years $(38-90+)$, with $74.4 \%$ male and $88.0 \%$ Caucasian (Table 1). In contrast, the median age of the overall study population at initiation of $1 \mathrm{~L}$ treatment was 72 years (31-90+), with 78.4\% male and 87.2\% Caucasian (Table 2 ). Additional baseline characteristics of the study population have been previously reported. ${ }^{20,21}$

At initiation of $1 \mathrm{~L}$ treatment, the baseline characteristics of the C-IO and C-C subgroups were similar (Table 1). The median hemoglobin level was lower among C-C patients (10.8 vs $12.2 \mathrm{~g} / \mathrm{dL}$ for $\mathrm{C}-\mathrm{IO}$ patients, 


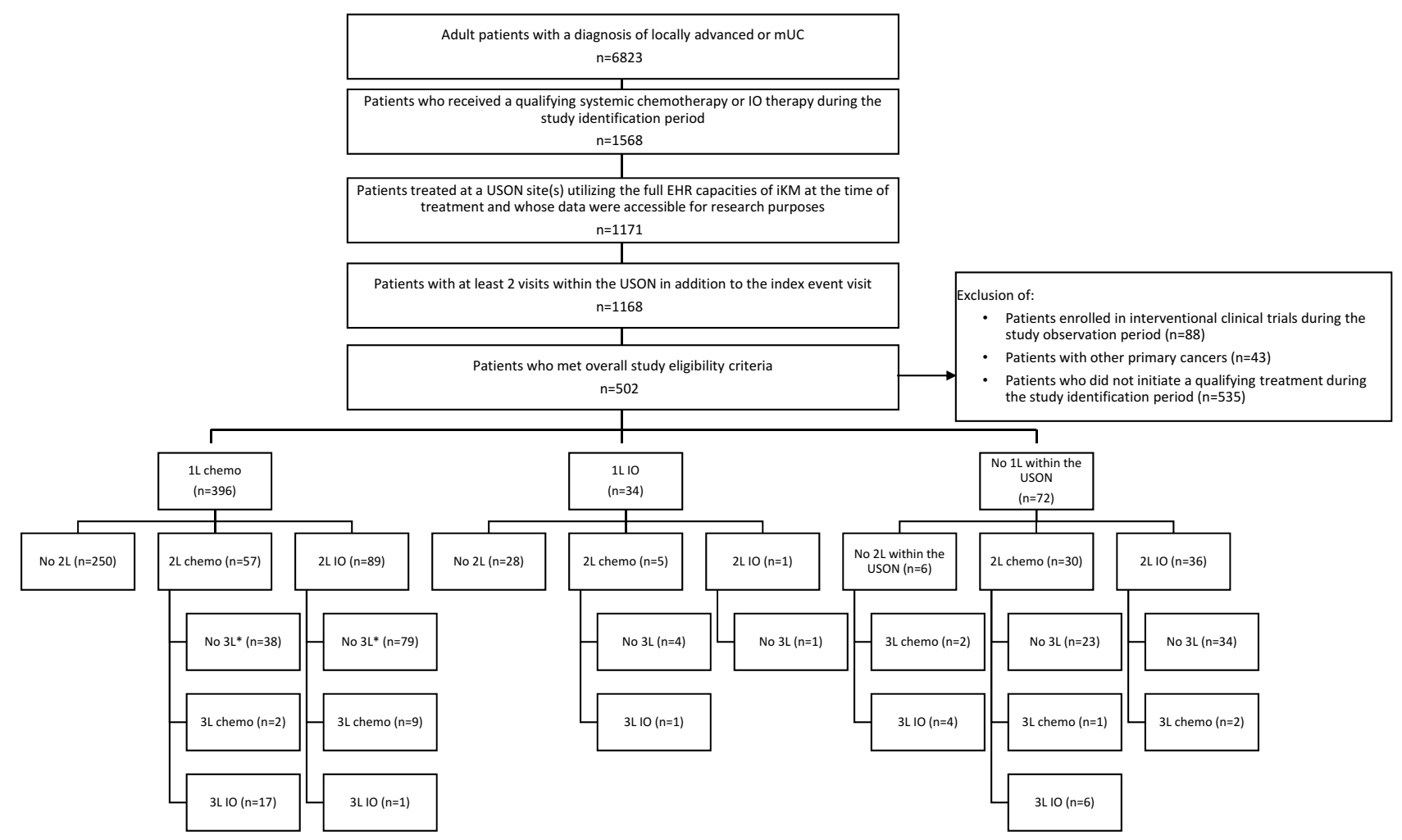

Figure I Study attrition.

Notes: $*$ These groups of patients were selected for the treatment sequencing subanalyses: patients who received IL chemotherapy, followed by either $2 \mathrm{~L}$ chemotherapy $(n=38)$ or IO-based regimens $(n=79)$ without a $3 \mathrm{~L}$ of treatment.

Abbreviations: IL, first-line treatment; $2 \mathrm{~L}$, second-line; 3L, third-line; chemo, chemotherapy; EHR, electronic health record; IO, immuno-oncology; iKM, iKnowMed; mUC, metastatic urothelial carcinoma; USON, US Oncology Network.

$\mathrm{P}=0.0091)$. The proportion of patients with aggressive disease status was numerically higher among patients who received the $\mathrm{C}-\mathrm{C}$ treatment sequence $(65.8 \%$ vs $58.2 \%$ in the $\mathrm{C}-\mathrm{IO}$ subgroup; $\mathrm{P}=0.7151$ ). Otherwise, no notable differences in demographic or clinical characteristics were observed. C-IO patients had a longer time from initiation of chemotherapy to start of IO therapy than those in the C-C subgroup had from initiation of $1 \mathrm{~L}$ chemotherapy to start of $2 \mathrm{~L}$ regimen (ie, median 6.7 vs 4.6 months, respectively; $\mathrm{P}=0.0047$ ).

\section{Clinical Outcomes}

Clinical outcomes for the overall study population are available in Table $3 .^{22}$

The median TTF from initiation of 1L treatment was 2.5 months (95\% CI 2.3-3.0) among patients who received the C-IO treatment sequence and 2.3 months $(95 \% \mathrm{CI}$ 1.4-3.7) among those who received the $\mathrm{C}-\mathrm{C}$ treatment sequence (unadjusted $\mathrm{HR}=0.832$ [95\% CI $0.562-1.232$ ]; HR $\mathrm{P}=0.3582$; Table 4; Figure 3). The median TFI was 2.2 months (95\% CI 1.6-3.5) among C-IO patients and 1.4 months (95\% CI 0.9-1.6) among the C-C subgroup (unadjusted HR 1.434 [95\% CI 0.965-2.130]; HR $\mathrm{P}=0.0742$ ). Lastly, the median OS was 19.2 months (95\% CI 11.9-not reported [NR]) among patients who received the C-IO treatment sequence and 11.9 months (95\% CI 8.5-20.8) among those who received the $\mathrm{C}-\mathrm{C}$ treatment sequence (unadjusted HR $0.627 \quad\left[\begin{array}{llll}95 \% & \text { CI } & 0.342-1.150\end{array}\right]$; HR $\mathrm{P}=0.1318)$.

\section{Discussion}

The baseline characteristics of the C-IO and C-C subgroups considered for analysis were similar to those in previously reported real-world $\mathrm{la} / \mathrm{mUC}$ patient populations. $^{23,24}$ In particular, Fisher et al (2018) performed a retrospective assessment of adult patients diagnosed with stage IV bladder cancer between 01 January 2008 and 01 June 2015 in US community oncology clinics. ${ }^{23}$ Among the 508 patients included in the analysis, the authors reported that the mean age was 70 years among a mostly male (75.2\%) and Caucasian (79.1\%) population. These demographic characteristics 


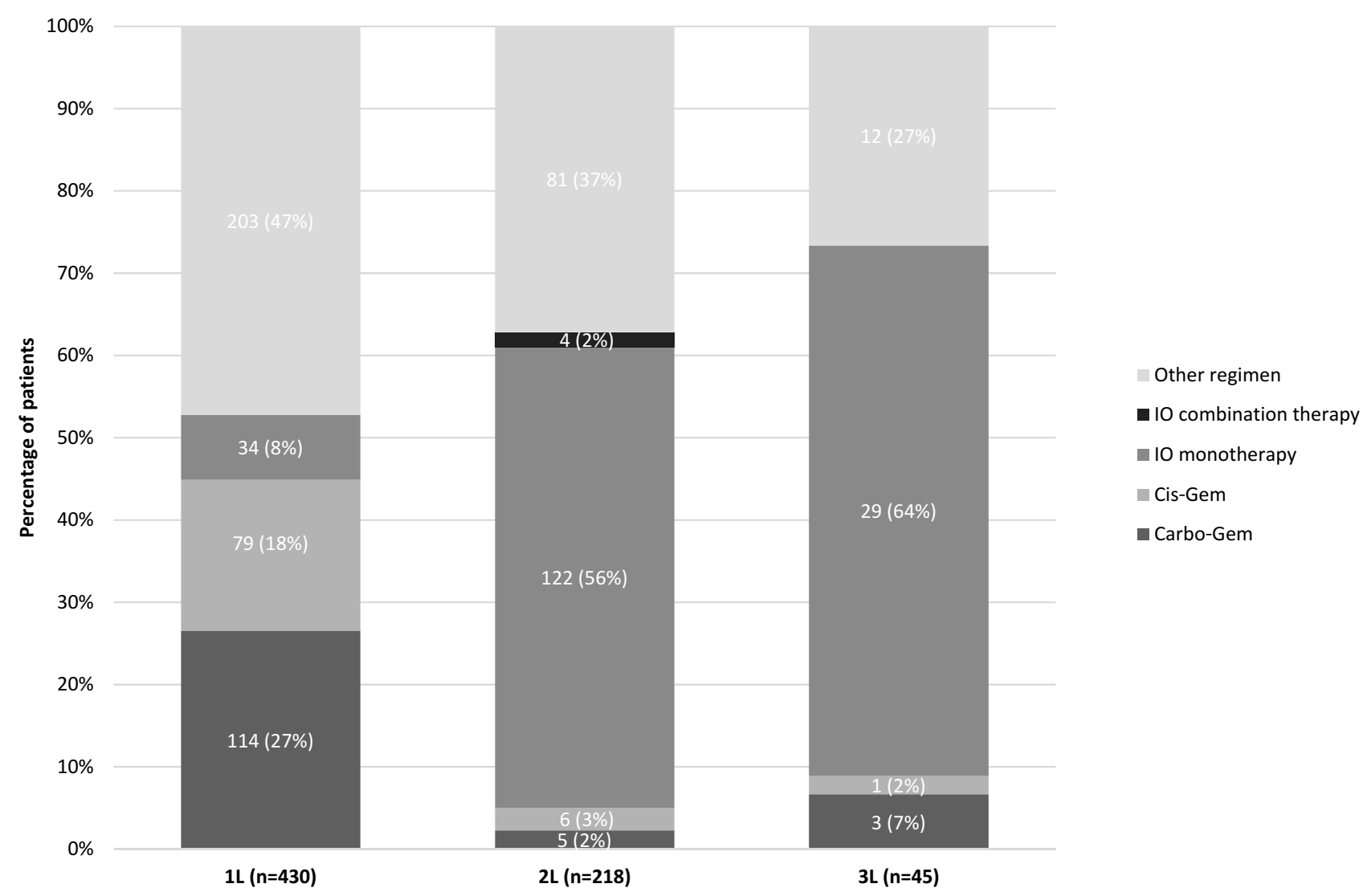

Figure 2 Regimen distribution by line of therapy.

Abbreviations: IL, first-line treatment; $2 \mathrm{~L}$, second-line; 3L, third-line; carbo-gem, carboplatin-gemcitabine; cis-gem, cisplatin-gemcitabine; IO, immuno-oncology.

are comparable to those observed in this study (median age of 69 years, with $74.4 \%$ male and $88.0 \%$ Caucasian) Likewise, Flannery et al (2019) reported that the median age of metastatic bladder cancer patients identified in the USON database between January 2010 and June 2014 was 72 years (range $26-90+$ ). ${ }^{24}$

Approximately $35 \%$ of $1 \mathrm{~L}$ patients in this study proceeded to a subsequent treatment. Previous studies have similarly reported high attrition rates across lines of therapy among la/mUC patients in real-world settings. Based on an analysis of the Surveillance, Epidemiology, and End Results (SEER)-Medicare database, Galsky et al (2018) reported that only $35 \%$ of advanced or metastatic bladder cancer patients who initiated $1 \mathrm{~L}$ treatment proceeded to a subsequent treatment. ${ }^{25}$ Similarly, Fisher et al found that 265 out of the $508(52 \%) 1 \mathrm{~L}$ patients included in their study received a next line of treatment. ${ }^{23}$ High attrition rates across lines of therapy suggest an unmet treatment need in this patient population, particularly for older and more frail patients who may not tolerate additional cytotoxic regimens.
It was observed that the time between initiation of the two treatments (time to $2 \mathrm{~L}$ ) was longer among $\mathrm{C}-\mathrm{IO}$ patients $(\mathrm{P}<0.001)$. This trend may have been influenced by the higher proportion of patients with aggressive disease in the $\mathrm{C}-\mathrm{C}$ subgroup relative to the $\mathrm{C}-\mathrm{IO}$ subgroup ( $65.8 \%$ vs $58.2 \%$, respectively, although this difference was not statistically significant). It was not possible, however, to determine if this difference in the proportion of patients with aggressive disease was due to underlying variation in patient characteristics or another factor, such as diagnostic testing requirements associated with use of IO regimens. In addition to the time to $2 \mathrm{~L}$ difference observed between groups, the median OS among patients who received the C-IO treatment sequence was 19.2 months, while the median OS was 11.9 months among those who received the $\mathrm{C}-\mathrm{C}$ treatment sequence.

Randomized clinical trials have investigated the role of IO therapies as a $1 \mathrm{~L}$ maintenance strategy among patients who respond to platinum-based $1 \mathrm{~L}$ treatment. ${ }^{16,17,26,27}$ The Phase III, open-label JAVELIN Bladder 100 trial evaluated the safety and efficacy of avelumab plus best supportive care 
Table I Demographic and Clinical Characteristics Among Treatment Sequence Subgroups

\begin{tabular}{|c|c|c|c|}
\hline & $\begin{array}{l}\text { Overall } \\
(n=117)\end{array}$ & $\begin{array}{l}\text { C-IO Subgroup } \\
(n=79)\end{array}$ & $\begin{array}{l}\text { C-C Subgroup } \\
(n=38)\end{array}$ \\
\hline Median age at IL treatment initiation (years; range) & $69(38,90+)$ & $71(40,90+)$ & $67(38,90+)$ \\
\hline Male - n (\%) & $87(74.4)$ & $60(75.9)$ & $27(71.1)$ \\
\hline \multicolumn{4}{|l|}{ Race - n (\%) } \\
\hline Caucasian & $103(88.0)$ & $66(83.5)$ & $37(97.4)$ \\
\hline Other/no information & $14(12.0)$ & $13(16.5)$ & I (2.6) \\
\hline \multicolumn{4}{|l|}{ Smoking status - n (\%) } \\
\hline Current & $23(19.7)$ & $14(17.7)$ & $9(23.7)$ \\
\hline Former & $60(51.3)$ & $40(50.6)$ & $20(52.6)$ \\
\hline Never & $29(24.8)$ & $20(25.3)$ & $9(23.7)$ \\
\hline No information & $5(4.3)$ & $5(6.3)$ & $0(0.0)$ \\
\hline \multicolumn{4}{|l|}{ ECOG performance score - $n(\%)$} \\
\hline 0 & $12(10.3)$ & $9(11.4)$ & $3(7.9)$ \\
\hline 1 & $67(57.3)$ & $44(55.7)$ & $23(60.5)$ \\
\hline 2 & $18(15.4)$ & II (I3.9) & $7(18.4)$ \\
\hline $3+$ & $2(1.7)$ & $\mathrm{I}(1.3)$ & I (2.6) \\
\hline No information & $18(15.4)$ & $14(17.7)$ & $4(10.5)$ \\
\hline \multicolumn{4}{|l|}{ Stage - n (\%) } \\
\hline 0 & $4(3.4)$ & $3(3.8)$ & I (2.6) \\
\hline 1 & $\mathrm{I}(0.9)$ & $\mathrm{I}(\mathrm{I} .3)$ & $0(0.0)$ \\
\hline II & $16(13.7)$ & $7(8.9)$ & $9(23.7)$ \\
\hline III & $6(5.1)$ & $4(5.1)$ & $2(5.3)$ \\
\hline IV & $66(56.4)$ & $48(60.8)$ & $18(47.4)$ \\
\hline No information & $24(20.5)$ & $16(20.3)$ & $8(21.1)$ \\
\hline Median ALT (U/L; range) & $18.0(7.0,44.0)$ & $17.0(7.0,44.0)$ & $18.5(7.0,42.0)$ \\
\hline Median AST (U/L; range) & $18.0(9.0,36.0)$ & $18.0(9.0,32.0)$ & $18.0(9.0,36.0)$ \\
\hline Median hemoglobin ( $\mathrm{g} / \mathrm{dL}$; range $)^{\mathrm{a}}$ & $11.6(7.6,13.7)$ & $12.2(7.7,13.7)$ & $10.8(7.6,13.7)$ \\
\hline Median creatinine clearance $\left(\mathrm{mL} / \mathrm{m}^{2} ;\right.$ range $)$ & $65.2(22.9,147.1)$ & $67.5(22.9,138.9)$ & $63.3(24.5,147.1)$ \\
\hline Median serum creatinine (mg/dL; range) & $1.2(0.6,2.0)$ & $1.2(0.7,2.0)$ & $1.2(0.6,1.9)$ \\
\hline \multicolumn{4}{|l|}{ Aggressive disease status - $\mathbf{n}(\%)$} \\
\hline No & $19(16.2)$ & $14(17.7)$ & $5(13.2)$ \\
\hline Yes & $71(60.7)$ & $46(58.2)$ & $25(65.8)$ \\
\hline No information & $27(23.1)$ & $19(24.1)$ & $8(21.1)$ \\
\hline Median disease free-interval (weeks; range) & $9.1(0.1,282.7)$ & II.I (0.I, 282.7) & $8.0(0.1,270.7)$ \\
\hline Median time from locally advanced/mUC diagnosis to IL initiation (weeks; range) & $2.3(0.1,266.9)$ & $2.3(0.1,103.3)$ & $2.4(0.1,266.9)$ \\
\hline Median time to $2 \mathrm{~L}$ (weeks; range) ${ }^{\mathrm{b}}$ & $24.9(0.1,102.1)$ & $29.0(4.1,102.1)$ & $20.0(0.1,63.1)$ \\
\hline \multicolumn{4}{|l|}{ Metastatic sites recorded - $n(\%)$} \\
\hline Patients with at least one recorded metastatic site & $68(58.1)$ & $45(57.0)$ & $23(60.5)$ \\
\hline Lung & $37(31.6)$ & $24(30.4)$ & $13(34.2)$ \\
\hline Bone & $23(19.7)$ & $13(16.5)$ & $10(26.3)$ \\
\hline Liver & $13(11.1)$ & $6(7.6)$ & $7(18.4)$ \\
\hline Retroperitoneum & $12(10.3)$ & $9(11.4)$ & $3(7.9)$ \\
\hline Other & $10(8.5)$ & $6(7.6)$ & $4(10.5)$ \\
\hline Mediastinum & $8(6.8)$ & $5(6.3)$ & $3(7.9)$ \\
\hline Peritoneum & $5(4.3)$ & $2(2.5)$ & $3(7.9)$ \\
\hline Brain & $4(3.4)$ & $3(3.8)$ & I (2.6) \\
\hline Skin & $2(1.7)$ & $2(2.5)$ & $0(0.0)$ \\
\hline
\end{tabular}

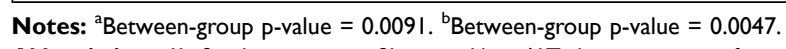

Abbreviations: IL, first-line treatment; $2 \mathrm{~L}$, second-line; ALT, alanine aminotransferase; AST, aspartate aminotransferase; C-C, first-line chemotherapy followed by second-line chemotherapy; C-IO, first-line chemotherapy followed by second-line immuno-oncology therapy; ECOG, Eastern Cooperative Oncology Group; IO, immuno-oncology; mUC, metastatic urothelial carcinoma. 
Table 2 Demographic and Clinical Characteristics at the Start of IL and 2L Therapy

\begin{tabular}{|c|c|c|c|}
\hline & Overall & $\begin{array}{l}\text { Systemic } \\
\text { Chemotherapies }\end{array}$ & $\begin{array}{l}10 \\
\text { Regimens }\end{array}$ \\
\hline \multicolumn{4}{|l|}{ At the start of IL treatment } \\
\hline Patients with available data - n (\%) & $430(100.0)$ & $396(92.1)$ & $34(7.9)$ \\
\hline Median age at IL treatment initiation (years; range) & $72(31,90+)$ & $72(31,90+)$ & $72(54,90+)$ \\
\hline Male - n (\%) & $337(78.4)$ & $312(78.8)$ & $25(73.5)$ \\
\hline \multicolumn{4}{|l|}{ Race - n (\%) } \\
\hline Caucasian & $375(87.2)$ & $345(87.1)$ & $30(88.2)$ \\
\hline Black or African American & $17(4.0)$ & $15(3.8)$ & $2(5.9)$ \\
\hline No information & $32(7.4)$ & $31(7.8)$ & I (2.9) \\
\hline Other & $6(1.4)$ & $5(1.3)$ & I (2.9) \\
\hline \multicolumn{4}{|l|}{ Smoking status - n (\%) } \\
\hline Current & $67(15.6)$ & $64(16.2)$ & $3(8.8)$ \\
\hline Former & $249(57.9)$ & $232(58.6)$ & $17(50.0)$ \\
\hline Never & $103(24.0)$ & $91(23.0)$ & $12(35.3)$ \\
\hline No information & II (2.6) & $9(2.3)$ & $2(5.9)$ \\
\hline \multicolumn{4}{|l|}{ ECOG performance score at IL initiation - $n$ (\%) } \\
\hline 0 & $52(12.1)$ & $48(12.1)$ & $4(11.8)$ \\
\hline 1 & $242(56.3)$ & $227(57.3)$ & $15(44.1)$ \\
\hline 2 & $61(14.2)$ & $55(13.9)$ & $6(17.6)$ \\
\hline $3+$ & $6(1.4)$ & $5(1.3)$ & I (2.9) \\
\hline No information & $69(16.0)$ & $61(15.4)$ & $8(23.5)$ \\
\hline \multicolumn{4}{|l|}{ Stage at diagnosis - $\mathbf{n}(\%)$} \\
\hline 0 & $10(2.3)$ & $9(2.3)$ & I (2.9) \\
\hline 1 & $16(3.7)$ & $15(3.8)$ & I (2.9) \\
\hline II & $91(21.2)$ & $86(21.7)$ & $5(14.7)$ \\
\hline III & $24(5.6)$ & $22(5.6)$ & $2(5.9)$ \\
\hline IV & $198(46.0)$ & $184(46.5)$ & $14(4 \mid .2)$ \\
\hline No information & $91(21.2)$ & $80(20.2)$ & II (32.4) \\
\hline \multicolumn{4}{|l|}{ Aggressive disease status $^{a}-n(\%)$} \\
\hline No & $86(20.0)$ & $75(18.9)$ & II (32.4) \\
\hline Yes & $260(60.5)$ & $246(62.1)$ & $14(4 \mid .2)$ \\
\hline No information & $84(19.5)$ & $75(18.9)$ & $9(26.5)$ \\
\hline $\begin{array}{l}\text { Median time from locally advanced/mUC diagnosis to IL initiation } \\
\text { (weeks; range) }\end{array}$ & $2.1(0.1,330.7)$ & $2.1(0.1,330.7)$ & $4.4(0.1,68.0)$ \\
\hline \multicolumn{4}{|l|}{ At the start of $2 \mathrm{~L}$ treatment } \\
\hline Patients with available data - n (\%) & $218(100.0)$ & $92(42.2)$ & $126(57.8)$ \\
\hline Median age at $2 \mathrm{~L}$ treatment initiation (years; range) & $69(39,90+)$ & $68(39,90+)$ & $69(4 I, 90+)$ \\
\hline Male - n (\%) & $166(76.1)$ & 71 (77.2) & $95(75.4)$ \\
\hline \multicolumn{4}{|l|}{ Race - n (\%) } \\
\hline Caucasian & $184(84.4)$ & $81(88.0)$ & $103(81.7)$ \\
\hline Black or African American & II (5.0) & $6(6.5)$ & $5(4.0)$ \\
\hline No information & $20(9.2)$ & $5(5.4)$ & $15(11.9)$ \\
\hline Other & $3(1.4)$ & $0(0.0)$ & $3(2.4)$ \\
\hline
\end{tabular}

(Continued) 
Table 2 (Continued).

\begin{tabular}{|c|c|c|c|}
\hline & Overall & $\begin{array}{l}\text { Systemic } \\
\text { Chemotherapies }\end{array}$ & $\begin{array}{l}\text { IO } \\
\text { Regimens }\end{array}$ \\
\hline \multicolumn{4}{|l|}{ Smoking status - n (\%) } \\
\hline Current & $32(14.7)$ & $14(15.2)$ & $18(14.3)$ \\
\hline Former & $113(51.8)$ & $51(55.4)$ & $62(49.2)$ \\
\hline Never & $62(28.4)$ & $23(25.0)$ & $39(31.0)$ \\
\hline No information & II (5.0) & $4(4.3)$ & $7(5.6)$ \\
\hline \multicolumn{4}{|l|}{ ECOG performance score at $2 \mathrm{~L}$ initiation - $n(\%)$} \\
\hline 0 & $13(6.0)$ & $3(3.3)$ & $10(7.9)$ \\
\hline I & II $8(54.1)$ & $49(53.3)$ & $69(54.8)$ \\
\hline 2 & $45(20.6)$ & $18(19.6)$ & $27(21.4)$ \\
\hline $3+$ & $\mathrm{I}(0.5)$ & $0(0.00)$ & I (0.8) \\
\hline No information & $4 \mathrm{I}(18.8)$ & $22(23.9)$ & $19(15.1)$ \\
\hline \multicolumn{4}{|l|}{ Stage at diagnosis - n (\%) } \\
\hline 0 & $4(1.8)$ & $\mathrm{I}(\mathrm{I} . \mathrm{I})$ & $3(2.4)$ \\
\hline I & $4(1.8)$ & $2(2.2)$ & $2(1.6)$ \\
\hline II & $33(15.1)$ & $17(18.5)$ & $16(12.7)$ \\
\hline III & $20(9.2)$ & $5(5.4)$ & $15(11.9)$ \\
\hline IV & $97(44.5)$ & $35(38.0)$ & $62(49.2)$ \\
\hline No information & $60(27.5)$ & $32(34.8)$ & $28(22.2)$ \\
\hline \multicolumn{4}{|l|}{ Aggressive disease status ${ }^{a}-n(\%)$} \\
\hline No & $31(14.2)$ & $14(15.2)$ & $17(13.5)$ \\
\hline Yes & $|2|(55.5)$ & $51(55.4)$ & $70(55.6)$ \\
\hline No information & $66(30.3)$ & $27(29.3)$ & $39(31.0)$ \\
\hline $\begin{array}{l}\text { Median time from locally advanced/mUC diagnosis to IL initiation } \\
\text { (weeks, range) }\end{array}$ & $2.4(0.1,330.7)$ & $2.4(0.1,330.7)$ & $2.4(0.1,103.3)$ \\
\hline
\end{tabular}

Notes: ${ }^{2}$ Aggressive disease was identified based on disease-free interval (time between diagnosis and presentation of locally advanced or metastatic disease < 12 months), pre-index performance status (ECOG $\geq 3$ ), and tumor grade (high-grade/grade 3/poorly differentiated). 'In the subset of patients who initiated $2 \mathrm{~L}$ treatment.

Abbreviations: IL, first-line treatment; 2L, second-line; 3L, third-line; ECOG, Eastern Cooperative Oncology Group; IO, immuno-oncology; mUC, metastatic urothelial carcinoma.

as $1 \mathrm{~L}$ maintenance therapy among la/mUC patients, regardless of PD-L1 status, whose disease did not progress with $1 \mathrm{~L}$ platinum-containing chemotherapy. ${ }^{14,16,17}$ At the first planned interim analysis, patients who received $1 \mathrm{~L}$ maintenance therapy with avelumab had significantly longer median OS than who received best supportive care alone. ${ }^{14}$ The results from this trial have led to avelumab becoming a recommended approach as maintenance therapy in patients whose disease does not progress on $1 \mathrm{~L}$ platinum-containing chemotherapy. ${ }^{3}$ Results from a Phase II randomized controlled trial of pembrolizumab (NCT02500121) vs placebo suggests that transitioning patients to an IO maintenance therapy following response on $1 \mathrm{~L}$ platinum-based treatment can extend PFS. ${ }^{28}$

Several prognostic factors, including performance status, sites of metastases, white blood cell counts, hemoglobin levels, and time from prior treatment, are positively associated with improved clinical outcomes among la/mUC patient populations. ${ }^{29-31}$ Similarly, the development of predictive biomarkers will be crucial to identify patients with la/mUC who respond to $1 \mathrm{~L}$ chemotherapy and are suitable for treatment with IO-based therapies in the 1L maintenance setting.

The results of this study should be considered in the context of the strengths and weaknesses of its design. As a retrospective observational study, treatment selection was based on clinical considerations, and underlying patient differences may have contributed to observed variation across groups, particularly for clinical outcomes. For example, physicians may have transitioned more frail patients and/or those experiencing toxicities to IO therapies for $2 \mathrm{~L}$ treatment. Additional research should be undertaken to understand the specific factors that influenced physician's choice of 
Table 3 Clinical Outcomes Across Lines of Therapy Among the Overall Study Population $(n=502)$

\begin{tabular}{|c|c|c|c|c|c|c|c|}
\hline & $\begin{array}{l}\text { Systemic } \\
\text { Chemotherapy } \\
\text { Restricted Mean (SE) }\end{array}$ & $\begin{array}{l}\text { IO Regimen } \\
\text { Restricted } \\
\text { Mean (SE) }\end{array}$ & $\begin{array}{l}\text { Systemic } \\
\text { Chemotherapy } \\
\text { Median (95\% Cl) }\end{array}$ & $\begin{array}{l}\text { IO Regimen } \\
\text { Median }(95 \% \\
\text { Cl) }\end{array}$ & $\begin{array}{l}\text { Log- } \\
\text { Rank } \\
\text { p-Value }\end{array}$ & $\begin{array}{l}\text { Unadjusted } \\
\text { Hazard Ratio } \\
(95 \% \mathrm{Cl})\end{array}$ & $\begin{array}{l}\text { Effect } \\
\text { p-Value }\end{array}$ \\
\hline \multicolumn{8}{|c|}{ Time to treatment failure (TTF; months) } \\
\hline IL & $\begin{array}{l}n=396 \\
3.6(0.2)\end{array}$ & $\begin{array}{l}n=34 \\
4.1(0.5)\end{array}$ & $2.4(2.3,2.7)$ & $3.0(2.3,5.8)$ & 0.0263 & $\begin{array}{l}0.621(0.404 \\
0.955)\end{array}$ & 0.0300 \\
\hline $2 \mathrm{~L}$ & $\begin{array}{l}\mathrm{n}=92 \\
3.0(0.3)\end{array}$ & $\begin{array}{l}n=126 \\
3.6(0.3)\end{array}$ & $2.1(1.5,2.8)$ & $3.1(2.1,4.9)$ & 0.0004 & $\begin{array}{l}0.568(0.412 \\
0.782)\end{array}$ & 0.0005 \\
\hline $3 \mathrm{~L}$ & $\begin{array}{l}n=16 \\
2.5(0.6)\end{array}$ & $\begin{array}{l}\mathrm{n}=29 \\
3.9(0.5)\end{array}$ & $2.3(0.3,4.3)$ & $5.8(1.6, N R)$ & 0.0371 & $\begin{array}{l}0.440(0.195 \\
0.995)\end{array}$ & 0.0487 \\
\hline \multicolumn{8}{|c|}{ Treatment-free interval (TFI; months) } \\
\hline $\mathrm{IL} \rightarrow 2 \mathrm{~L}$ & $\begin{array}{l}n=370 \\
7.0(0.4)\end{array}$ & $\begin{array}{l}n=22 \\
7.0(0.4)\end{array}$ & $4.1(3.2,5.2)$ & $1.3(0.6,2.0)$ & $<0.0001$ & $\begin{array}{l}4.007(2.348 \\
6.836)\end{array}$ & $<0.0001$ \\
\hline \multicolumn{8}{|c|}{ Overall survival (OS; months) } \\
\hline IL & $\begin{array}{l}n=396 \\
I 5 . \mid(0.5)\end{array}$ & $\begin{array}{l}n=34 \\
9.2(1.1)\end{array}$ & $16.4(13.4,18.9)$ & I $3.0(4.3, N R)$ & 0.0607 & $\begin{array}{l}\text { I.84I (0.963, } \\
3.521)\end{array}$ & 0.0649 \\
\hline $2 \mathrm{~L}$ & $\begin{array}{l}\mathrm{n}=92 \\
11.7(0.9)\end{array}$ & $\begin{array}{l}n=126 \\
7.1(0.5)\end{array}$ & II.8 (7.9, NR) & $6.8(4.9,9.6)$ & 0.0567 & $\begin{array}{l}\text { I.544 (0.984, } \\
2.422)\end{array}$ & 0.0587 \\
\hline $3 \mathrm{~L}$ & $\begin{array}{l}n=16 \\
4.2(0.7)\end{array}$ & $\begin{array}{l}n=29 \\
5.5(0.5)\end{array}$ & $3.9(\mathrm{I} .3, \mathrm{NR})$ & $6.7(3.9, N R)$ & 0.2347 & $\begin{array}{l}0.556(0.208 \\
\text { I.484) }\end{array}$ & 0.2413 \\
\hline
\end{tabular}

Abbreviations: IL, first-line; $2 \mathrm{~L}$, second-line; 3L, third-line; $\mathrm{Cl}$, confidence interval; $\mathrm{OO}$, immuno-oncology; NR, not reached; SE, standard error.

Table 4 Clinical Outcomes by Most Common Treatment Sequences

\begin{tabular}{|c|c|c|c|c|}
\hline & \multirow{2}{*}{$\begin{array}{l}\text { C-IO } \\
(n=79)\end{array}$} & \multirow{2}{*}{$\begin{array}{l}\text { C-C } \\
(n=38)\end{array}$} & \multirow[t]{2}{*}{ Unadjusted Hazard Ratio ${ }^{a}(95 \% \mathrm{Cl})$} & \multirow[t]{2}{*}{ Hazard Ratio p-Value } \\
\hline & & & & \\
\hline Median (95\% Cl) TTF (months) & $2.5(2.3,3.0)$ & $2.3(1.4,3.7)$ & $0.832(0.562,1.232)$ & 0.3582 \\
\hline Restricted mean (SE) TTF (months) & $3.7(0.3)$ & $3.3(0.4)$ & & \\
\hline Median $(95 \% \mathrm{Cl}) \mathrm{TFI}$ (months) & $2.2(1.6,3.5)$ & $\mathrm{I} .4(0.9,1.6)$ & $1.434(0.965,2.130)$ & 0.0742 \\
\hline Restricted mean (SE) TFI (months) & $3.6(0.4)$ & $2.6(0.6)$ & & \\
\hline Median $(95 \% \mathrm{Cl})$ OS (months) & $19.2(11.9, N R)$ & $11.9(8.5,20.8)$ & $0.627(0.342,1.150)$ & 0.1318 \\
\hline Restricted mean (SE) OS (months) & $14.7(0.7)$ & I3.4 (I.3) & & \\
\hline
\end{tabular}

Notes: ${ }^{a}$ Unadjusted hazard ratios and hazard ratio $\mathrm{p}$-values are based on univariate Cox regression analyses.

Abbreviations: C-C, first-line chemotherapy followed by second-line chemotherapy; $\mathrm{Cl}$, confidence interval; C-IO, first-line chemotherapy followed by second-line immuno-oncology therapy; NR, not reached; OS, overall survival; TFI, treatment-free interval; TTF, time to treatment failure; SE, standard error.

treatment sequence and how these may change with expanded approvals of IO-based therapies.

As PD-L1 expression testing was not routinely performed in clinical practice at the time of study, we were unable to determine how PD-L1 tumor expression levels or PD-L1 testing methods affected the choice of therapy and patient outcomes. Over time, PD-L1 expression testing may become more widespread in clinical practice, and future studies should consider how expression levels influence outcomes.
Study data were sourced from the USON's EHR, $\mathrm{iKM}$, which was originally populated for clinical practice purposes, not research. Consequently, there may have been data entry errors or missing values that introduced some level of misclassification bias, and some variables of interest were not complete across the study population. Cost data were unavailable for healthcare resource use assessments and, as such, it was not possible to evaluate the direct and indirect economic implications of the treatment sequences. Lastly, the 


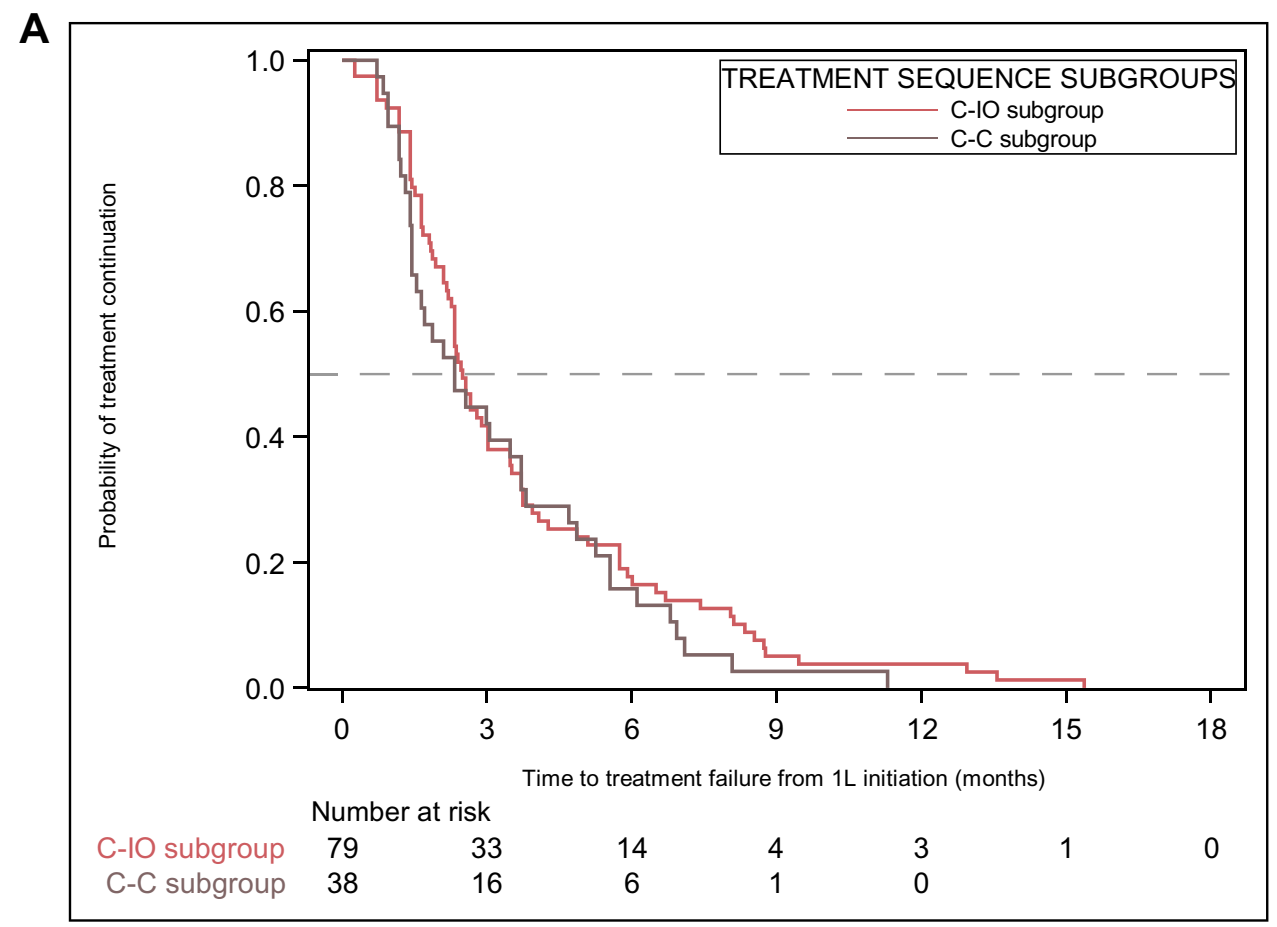

B

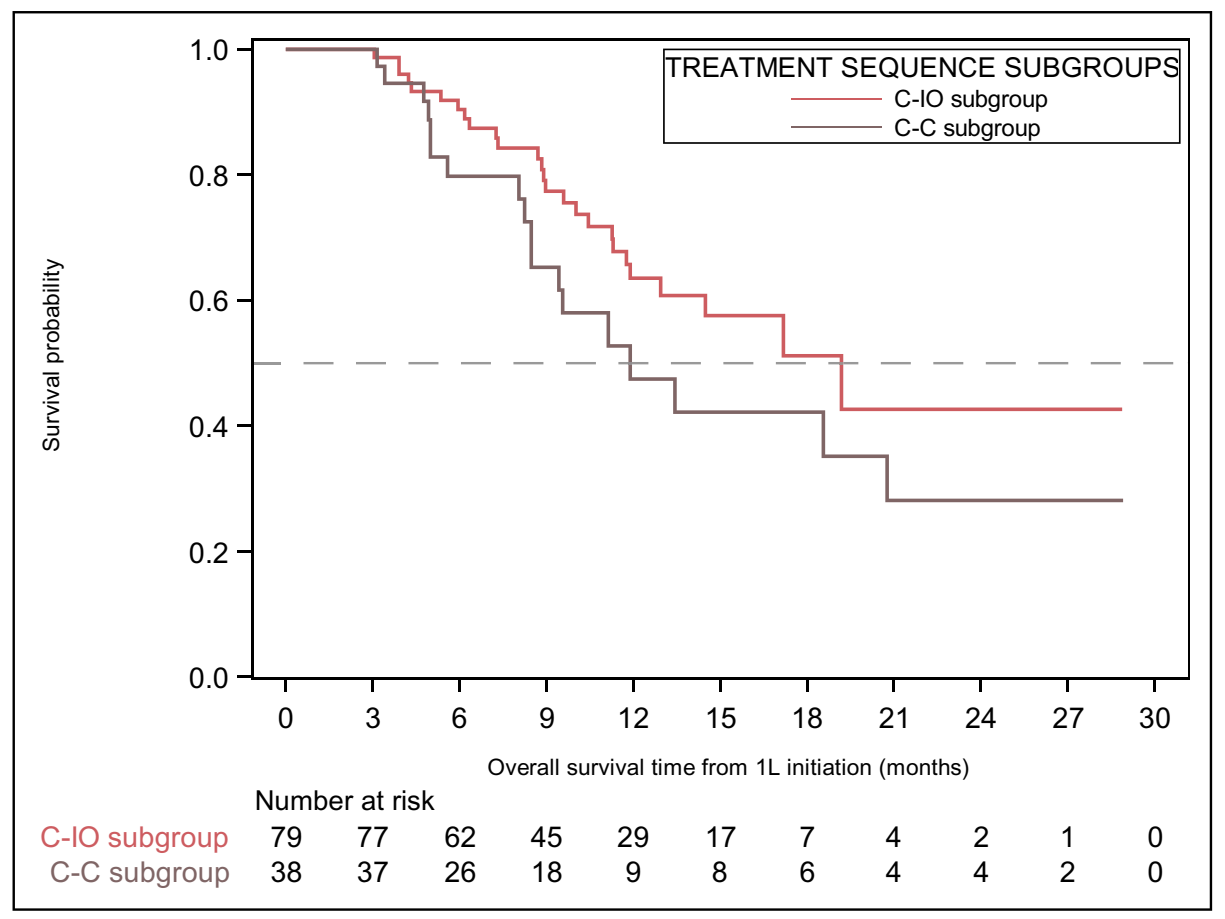

Figure 3 Kaplan-Meier curves for clinical outcomes by treatment sequence.

Notes: Time to treatment failure $(\mathbf{A})$ and overall survival $(\mathbf{B})$.

Abbreviations: C-C, first-line chemotherapy followed by second-line chemotherapy; C-IO, first-line chemotherapy followed by second-line immuno-oncology therapy.

results of this study may not be generalizable to community oncology clinics outside the USON or those within the USON that do not utilize the full EHR capabilities.

\section{Conclusions}

This study provides real-world insight into treatment sequences and clinical outcomes of $\mathrm{la} / \mathrm{mUC}$ patients who received care in US community oncology clinics as IO 
therapies were being adopted into clinical practice. Consistent with treatment guidelines, community oncology clinics appear to be adopting IO-based regimens as a subsequent treatment as the standard of care. As reported by other real-world studies, high attrition rates across lines of therapy were observed, suggesting an unmet need in this patient population. This study found notable improvements in OS among patients who received the C-IO treatment sequence versus those who received the C-C treatment sequence. As further evidence from ongoing trials of IO therapies emerges, including the potential role of $1 \mathrm{~L}$ maintenance approach among patients who respond to platinum-based chemotherapy, it will be important to consider how these trends and outcomes manifest in the real-world community oncology setting. The results of this study may be used as a benchmark for these future studies.

\section{Abbreviations}

1L, first-line; 2L, second-line; 3L, third-line; ALT, alanine aminotransferase; ANOVA, analysis of variance; AST, aspartate aminotransferase; carbo-gem, carboplatingemcitabine; C-C, first-line chemotherapy followed by second-line chemotherapy; chemo, chemotherapy; CI, confidence interval; C-IO, first-line chemotherapy followed by second-line immuno-oncology therapy; cisgem, cisplatin-gemcitabine; DMF, Death Master File; ECOG, Eastern Cooperative Oncology Group; EHR, electronic health record; HR, hazard ratio; iKM, iKnowMed; IO, immuno-oncology; la/mUC, locally advanced or metastatic urothelial carcinoma; NR, not reached; OS, overall survival; PD-1, programmed cell death 1 protein; PD-L1, programmed cell death 1 ligand 1; PFS, progression-free survival; SE, standard error; SEER, Surveillance, Epidemiology, and End Results; SPEAR-Bladder, Study informing treatment Pathway dEcision in bladder cAnceR; TFI, treatment-free interval; TTF, time to treatment failure; USON, US Oncology Network.

\section{Data Sharing Statement}

The health data used to support the findings of this study are restricted by the US Oncology Institutional Review Board in order to protect patient privacy. For this reason, data used to support the findings of this study have not been made available.

\section{Ethics Approval and Informed Consent}

The study protocol was granted an exception and waiver of informed consent by the US Oncology Institutional Review Board.

\section{Funding}

This study was sponsored by EMD Serono, Inc.; an affiliate of Merck KGaA, Darmstadt, Germany, as part of an alliance between Merck KGaA and Pfizer.

\section{Disclosure}

A Bhanegaonkar and M Bharmal are employees of EMD Serono Research \& Development Institute, Inc., Rockland, MA, USA; an affiliate of Merck KGaA, Darmstadt, Germany. M Kearney is an employee of Merck KGaA, Darmstadt, Germany. P Cislo and R Kim are employees of Pfizer, New York, NY, USA. M Boyd and KM Aguilar are employees of McKesson Life Sciences, The US Oncology Network, The Woodlands, TX, USA. H Phatak was an employee of EMD Serono Research \& Development Institute, Inc., Rockland, MA, USA; an affiliate of Merck KGaA, Darmstadt, Germany, at the time the study was conducted. The authors have no other relevant affiliations or financial involvement with any organization or entity with a financial interest in or financial conflict with the subject matter or materials discussed in the manuscript apart from those disclosed. Representatives from these organizations provided oversight to the study design, project execution, interpretation of data, and writing of the report.

\section{References}

1. Narayanan S, Harshman LC, Srinivas S. Second-line therapies in metastatic urothelial carcinoma. Hematol Oncol Clin North Am. 2015;29(2):341-359. doi:10.1016/j.hoc.2014.10.007

2. NCCN Guidelines for Bladder Cancer V3.2019. Clinical practice guidelines in oncology: bladder cancer version 3.2019. CNational Comprehensive Cancer Network, Inc. 2019. All rights reserved. NCCN.org. Available from: https://www.nccn.org/professionals/physi cian_gls/pdf/bladder.pdf. Accessed May 9, 2019.

3. NCCN Guidelines for Bladder Cancer V6.2020. Clinical practice guidelines in oncology: bladder cancer version 6.2020. Available from: https://www.nccn. org/professionals/physician_gls/pdf/bladder.pdf. Accessed August 19, 2020.

4. Bukhari N, Al-Shamsi HO, Azam F. Update on the Treatment of Metastatic Urothelial Carcinoma. Scientific World Journal. 2018;2018:1-7. doi:10.1155/2018/5682078

5. von der Maase H, Sengelov L, Roberts JT, et al. Long-term survival results of a randomized trial comparing gemcitabine plus cisplatin, with methotrexate, vinblastine, doxorubicin, plus cisplatin in patients with bladder cancer. $J$ Clin Oncol. 2005;23(21):4602-4608. doi:10.1200/JCO.2005.07.757 
6. De Santis M, Bellmunt J, Mead G, et al. Randomized phase II/III trial assessing gemcitabine/carboplatin and methotrexate/carboplatin/vinblastine in patients with advanced urothelial cancer who are unfit for cisplatin-based chemotherapy: EORTC study 30986. J Clin Oncol. 2012;30(2):191-199. doi:10.1200/JCO.2011.37.3571

7. Bellmunt J, Theodore C, Demkov T, et al. Phase III trial of vinflunine plus best supportive care compared with best supportive care alone after a platinum-containing regimen in patients with advanced transitional cell carcinoma of the urothelial tract. J Clin Oncol. 2009;27 (27):4454-4461. doi:10.1200/JCO.2008.20.5534

8. Kim HS, Seo HK. Immune checkpoint inhibitors for urothelial carcinoma. Investig Clin Urol. 2018;59(5):285-296. doi:10.4111/ icu.2018.59.5.285

9. Di Nunno V, De Luca E, Buttigliero C, et al. Immune-checkpoint inhibitors in previously treated patients with advanced or metastatic urothelial carcinoma: A systematic review and meta-analysis. Crit Rev Oncol Hematol. 2018;129:124-132. doi:10.1016/j.critrevonc.2018.07.004

10. Inman BA, Sebo TJ, Frigola X, et al. PD-L1 (B7-H1) expression by urothelial carcinoma of the bladder and BCG-induced granulomata: associations with localized stage progression. Cancer. 2007;109 (8):1499-1505. doi:10.1002/cncr.22588

11. Postow MA, Callahan MK, Wolchok JD. Immune Checkpoint Blockade in Cancer Therapy. J Clin Oncol. 2015;33(17):19 74-1982. doi:10.1200/JCO.2014.59.4358

12. Bellmunt J, Mullane SA, Werner L, et al. Association of PD-L1 expression on tumor-infiltrating mononuclear cells and overall survival in patients with urothelial carcinoma. Ann Oncol. 2015;26 (4):812-817. doi:10.1093/annonc/mdv009

13. Faraj SF, Munari E, Guner G, et al. Assessment of tumoral PD-L1 expression and intratumoral CD8+ $\mathrm{T}$ cells in urothelial carcinoma. Urology. 2015;85(3):703e701-706. doi:10.1016/j.urology.2014.10.020

14. Powles T, Park SH, Voog E, et al. Avelumab maintenance therapy for advanced or metastatic urothelial carcinoma. $N$ Engl J Med.2020;383 (13):1218-1230.doi:10.1056/NEJMoa2002788

15. Bellmunt J, Orsola A, Leow JJ, Wiegel T; Group EGW. eUpdate Bladder Cancer Treatment Recommendations; 2020. Available from: https://www.esmo.org/guidelines/genitourinary-cancers/bladdercancer/eupdate-bladder-cancer-treatment-recommendations4. Accessed August 27, 2020.

16. EMD Serono Inc. and Pfizer Inc. Bavencio (avelumab) injection, for intravenous use. Prescribing Information. Rockland, MA; June 2020. Available from: https://www.emdserono.com/us-en/pi/bavencio-pi. pdf. Accessed August 20, 2020.

17. ClinicalTrials.gov. A Study of Avelumab in Patients with Locally Advanced or Metastatic Urothelial Cancer (JAVELIN Bladder 100; NCT02603432); 2018. Available from: https:/clinicaltrials.gov/ct2/ show/NCT02603432. Accessed May 17, 2019.

18. Powles T, Park SH, Voog E, Caserta C, Valderrama BP, Gurney H. Maintenance avelumab + best supportive care (BSC) versus BSC alone after platinum-based first-line (1L) chemotherapy in advanced urothelial carcinoma (UC): JAVELIN Bladder 100 phase III interim analysis. J Clin Oncol. 2020;38 (18_suppl):LBA1. doi:10.1200/JCO.2020.38.18_suppl.LBA1
19. McKesson. The US Oncology Network; 2019. Available from: https://www.usoncology.com/our-company. Accessed May 16, 2019.

20. Doshi G, Bhanegaonkar A, Bharmal M, et al. Patient characteristics and treatment patterns from SPEAR-BLADDER: Study informing treatment Pathway dEcision in bladder cAnceR. Value Health. 2018;21(1):S49. doi:10.1016/j.jval.2018.04.290

21. Postow MA, Callahan MK, Wolchok JD. Immune Checkpoint Blockade in Cancer Therapy. J Clin Oncol. 2015;10;33(17):19741982. doi:10.1200/JCO.2014.59.4358

22. Doshi G, Bhanegaonkar A, Bharmal M, et al. SPEAR-Bladder (Study informing treatment Pathway dEcision in bladder cAnceR): firstthrough third-line time to treatment failure in the US. J Clin Oncol. 2018;36(15_suppl):4544. doi:10.1200/JCO.2018.36.15_suppl.4544

23. Fisher MD, Shenolikar R, Miller PJ, Fenton M, Walker MS. Treatment patterns and outcomes in stage IV bladder cancer in a community oncology setting: 2008-2015. Clin Genitourin Cancer. 2018;16(6):e1171-e1179. doi:10.1016/j.clgc.2018.07.025

24. Flannery K, Boyd M, Black-Shinn J, Robert N, Kamat AM. Outcomes in patients with metastatic bladder cancer in the USA: a retrospective electronic medical record study. Future Oncol. 2019;15(12):1323-1334. doi:10.2217/fon-2018-0654

25. Galsky MD, Pal SK, Lin SW, et al. Real-world effectiveness of chemotherapy in elderly patients with metastatic bladder cancer in the United States. Bladder Cancer. 2018;4(2):227-238. doi:10.3233/BLC-170149

26. Galsky MD, Pal SK, Mortazavi A, et al. Randomized double-blind Phase II study of maintenance pembrolizumab versus placebo after first-line chemotherapy in patients (pts) with metastatic urothelial cancer (mUC): HCRN GU14-182. J Clin Oncol. 2019;37 (15_suppl):4504. doi:10.1200/JCO.2019.37.15_suppl.4504

27. ClinicalTrials.gov. Testing the PD-1 Inhibitor Pembrolizumab as Maintenance Therapy After Initial Chemotherapy in Metastatic Bladder Cancer (NCT02500121); 2018. Available from: https://clin icaltrials.gov/ct2/show/NCT02500121. Accessed May 17, 2019.

28. Galsky MD, Mortazavi A, Milowsky MI, et al. Randomized double-blind phase II study of maintenance pembrolizumab versus placebo after first-line chemotherapy in patients with metastatic urothelial cancer. J Clin Oncol. 2020;38(16):1797-1806. doi:10.12 00/JCO.19.03091

29. Galsky MD, Moshier E, Krege S, et al. Posttreatment prognostic nomogram for patients with metastatic urothelial cancer completing first-line cisplatin-based chemotherapy. Urol Oncol. 2014;32 (1):48e41-48. doi:10.1016/j.urolonc.2013.07.001

30. Sonpavde G, Pond GR, Fougeray R, et al. Time from prior chemotherapy enhances prognostic risk grouping in the second-line setting of advanced urothelial carcinoma: a retrospective analysis of pooled, prospective phase 2 trials. Eur Urol. 2013;63(4):717-723. doi:10.1016/j.eururo.2012.11.042

31. Matsumoto R, Abe T, Ishizaki J, et al. Outcome and prognostic factors in metastatic urothelial carcinoma patients receiving second-line chemotherapy: an analysis of real-world clinical practice data in Japan. Jpn J Clin Oncol. 2018;48(8):771-776. doi:10.1093/jjco/hyy094
ClinicoEconomics and Outcomes Research

\section{Publish your work in this journal}

ClinicoEconomics and Outcomes Research is an international, peerreviewed open-access journal focusing on Health Technology Assessment, Pharmacoeconomics and Outcomes Research in the areas of diagnosis, medical devices, and clinical, surgical and pharmacological intervention. The economic impact of health policy and health systems organization also constitute important areas of coverage. The manuscript management system is completely online and includes a very quick and fair peer-review system, which is all easy to use. Visit http://www.dovepress.com/testimonials.php to read real quotes from published authors. 